\title{
Mouse killing or carrying by male and female Long-Evans hooded rats*
}

\author{
DANIEL J. LONOWSKI, ROBERT A. LEVITT \\ and \\ SCOTT D. LARSON \\ Southern Illinois University, Carbondale, Ill. 62901
}

The behavioral responses of rats toward mice were studied. A portion of the rats killed the mice, as found in previous studies. Another group of rats exhibited a different, previously unstudied response of mouse carrying (without the kill). A behavioral study of the stability of mouse killing or carrying was carried out. The behaviors were found to be stable over two trials in 1 day and over 5 consecutive days of testing. The possible behavioral significance of the carrying response was also discussed.

Predatory behavior can be elicited in laboratory rats by several species of test animals. The effectiveness of a given prey object, however, in evoking a predatory response by a rat may vary considerably. In two species of rats tested, almost all killed frogs and turtles; whereas chicks elicited about 50\% kills and mice 10\%-20\% kills (Bandler \& Moyer, 1970).

Among the effective prey objects studied, considerable attention has been given to the mouse as a test stimulus. Rats that do kill have been shown to engage consistently in mouse killing for many months when tested under standard environmental conditions (Karli, Vergnes, \& Didiergeorges, 1969), and the overall response topography satisfactorily conforms to the criteria that define predatory aggression (Moyer, 1968). The predatory attack by a laboratory rat directed at a mouse is typically highly stereotyped. Upon presentation of a mouse, a rat will quietly pounce upon and seize its prey, curl its back in an upward direction while holding the mouse with all four paws, and, with successive bites in the cervical region of the spine, kill the mouse (Karli, 1956).

Despite the high degree of predictability for the occurrence of mouse killing, the majority of rats (80\%) do not exhibit this behavior. Nonkiller rats may even starve to death in the presence of a live mouse without killing it, despite the mouse's potential food value (Karli, 1956). From this general observation, it has been thought that nonkiller rats are a homogeneous class of animals with regard to their behavior in the presence of mice. Among nonkiller rats, however, are a distinct group of animals that exhibit a form of behavior as dynamic in its own right as mouse killing. This behavior is mouse carrying.

\footnotetext{
*Supported in part by USPHS Grant MH-14381.
}

Mouse carrying is typified by a rat quickly seizing a mouse with its teeth near the cervical spine, picking up the mouse, and carrying it to a distant area of the cage. Preliminary results in this laboratory reveal that, in a period of $15 \mathrm{~min}$, a rat may carry a mouse from 1 to 65 times, with an average of 9 carries. Furthermore, approximately $20 \%$ of nonkiller rats of either sex will engage in this form of behavior.

The physiological basis of predatory behavior in the laboratory rat has in recent years been extensively studied. The elicitation of mouse killing in nonkiller rats by electrical brain stimulation (King \& Hoebel, 1968; Panksepp, 1971; Vergnes \& Karli, 1969) and the differential elicitation and inhibition of mouse killing by cholinergic brain stimulation (Smith, King, \& Hoebel, 1970; Bandler, 1969, 1970, 1971a, b) have clearly shown involvement of limbic system structures in the mediation of mouse-killing behavior.

During the course of any experiment designed to manipulate the natural expression of an animal's behavior, a careful and efficient means of insuring response stability must be demonstrated. A stable kill latency, as defined by Smith, King, \& Hoebel (1968), is described as a kill occurring within 2 min on any 3 successive days of testing within a total test period of 17 days. Kill response stabilization may also be defined statistically, as done by Bandler $(1969,1970,1971 \mathrm{a}, \mathrm{b})$, where kill latencies were deemed normalized when they did not vary significantly for 3 days in a total test period of 9 days.

The possible loss of valuable experimental time and effort, in light of the methods employed in the past, raises the possibility that a more efficient and effective method for establishing a stable response level in killer rats might be found. We now report results of an experiment which investigated two methods to achieve this end. Furthermore, mouse carrying was studied in a like manner, since neurological investigations of this new phenomenon may answer many questions still surrounding the behavior of rats presented with mice. This is, to our knowledge, the first investigation of the mouse-carrying phenomenon. Our observations consist of comparative analyses of male and female killer and carrier rats when tested on two trials per day for 5 successive days.

\section{METHOD}

Twenty-five male and 25 female adult Long-Evans hooded rats served as experimental Ss. Each animal weighed between 250-350 g at the start of the experiment. All rats were housed individually, with food and water available ad lib. 


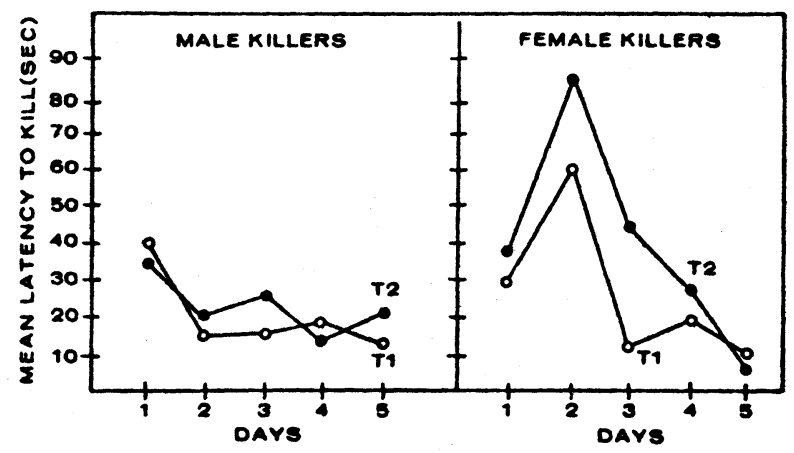

Fig. 1. Latency to kill on Trials 1 and 2 over 5 test days for male and female killer rats.

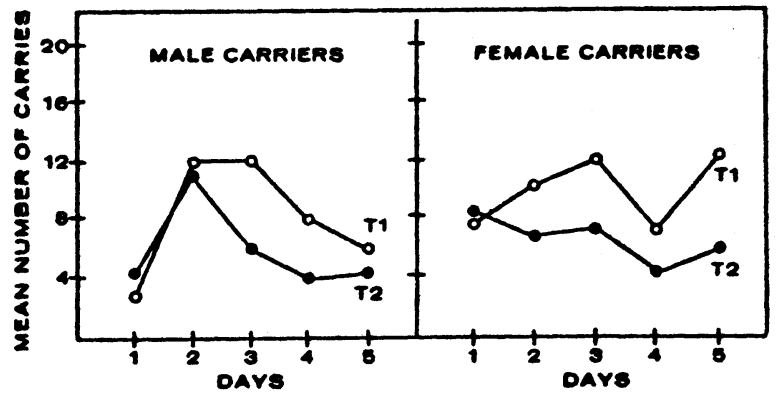

Fig. 2. Number of carries on Trials 1 and 2 over 5 test days for male and female carrier rats.

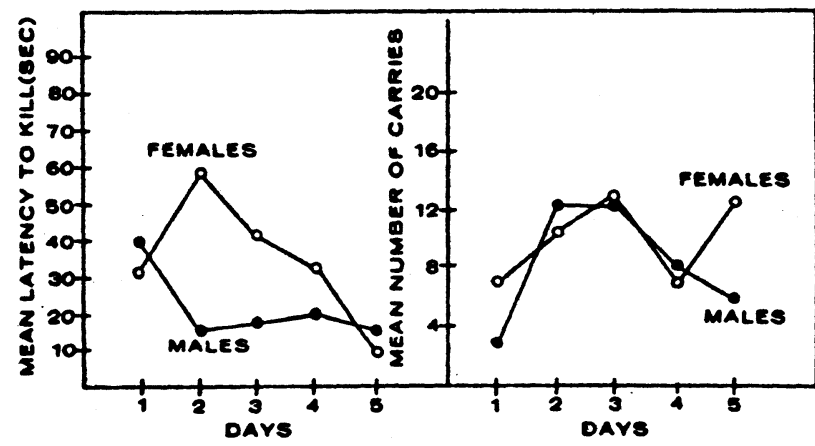

Fig. 3. Latency to kill or number of carries on Trial 1 for 5 test days in male and female rats.

A behavioral classification of killer or carrier was made in the following manner: Each rat was taken from its home cage, held gently in hand for $10-20 \mathrm{sec}$, then returned to its cage (this procedure was required, since experimental treatments with these animals often require handling just prior to behavioral testing). Following a 5-min interval, which represented a hypothetical drug-onset time, a naive albino mouse was placed in the rat's cage. The amount of time elapsing until the kill or the number of carries by killers or carriers, respectively, was recorded during a 15-min test session. At the end of the first 15-min trial, the mouse (living or dead) was removed from the test cage and $30 \mathrm{~min}$ were allowed to pass. A second trial was then begun in the same manner as outlined above. Thus, each rat received two 15 -min trials per day (separated by a 30 -min rest period) for 5 successive days of testing.

\section{RESULTS}

Of the 50 rats initially selected ( 25 males and 25 females), 8 male and 5 female killers and 4 male and 5 female carriers were obtained. The remaining 28 rats were neither killers nor carriers and were not included in statistical analyses.

Figures 1 and 2 show the results of testing the killers and carriers of each sex for two trials on each of 5 consecutive days. Following a tabulation of all data, an analysis of variance indicated no significant differences in latency to kill or in the number of carries during the 15-min trials. This can be seen in Figs. 1 and 2, where it is evident that neither latency to kill (Fig. 1) nor number of carries (Fig. 2) on Trials 1 and $2\left(T_{1}\right.$ and $T_{2}$ ) were markedly variant within days $\left(T_{1}\right.$ vs $\left.T_{2}\right)$ or across days (Days 1-5). Furthermore, a comparison of these responses for male and female killers and carriers across days was not dissimilar. This result is graphically illustrated for the $T_{1}$ data in Fig. 3.

\section{DISCUSSION}

This experiment represents an attempt to ascertain an efficient and reliable method for selecting Ss that exhibit a stable mouse-killing or mouse-carrying response. Studies which propose to manipulate selectively a rat's behavior in response to mice by neurological techniques must first demonstrate that a stable response level has been achieved in each $\mathrm{S}$. These results indicate that a procedure to meet this experimental requirement is accessible.

Since no statistically significant response differences were found from trial to trial or day to day, experimental treatments may be introduced in mouse killing or carrying studies on a second trial within a single day and compared to control behavior on the first trial, or on any day following the first day of testing, when one trial is conducted each day and compared to previous control observations.

The significance of mouse killing as a form of predatory aggression has been firmly established since the initial observations by Karli (1956) and later by the behavioral criteria used to define predatory aggression in the rat (Moyer, 1968).

This study introduces the phenomenon of natural mouse carrying. Rats that carry but do not kill mice, although previously viewed as behaviorally static animals, must now be considered as behaviorally dynamic. For this reason, carrier rats should not be included in a general class of nonkiller rats; nor should they be excluded from careful behavioral scrutiny, as has been the case in the past.

Why some rats kill while others carry is far from clear. However, two explanations may be provided that seem plausible: Since the overall behavioral pattern of a carrying response is almost identical to the stereotyped killing response, except for the killing bite, one might be led to suspect that carrying represents an incomplete form of predatory aggression in the laboratory rat.

In contrast, mouse carrying by rats may fall into a response category distinct from predatory aggression. Since female rats are known to carry and retrieve rat pups, carrying by female as well as by male adult rats may be similar to a kind of maternal or paternal response.

The etiology of mouse carrying, although open to conjecture, may very well help to explain the dichotomy of killers and traditionally defined nonkiller rats. It should also be pointed out that by no means are all rats either mouse killers or mouse carriers. In this study, $56 \%$ of the rats tested were neither killers nor carriers.

\section{REFERENCES}

Bandler, R. J., Jr. Facilitation of aggressive behavior in the rat by 
direct cholinergic stimulation of the hypothalamus. Nature, $1969,224,1035-1036$.

Bandler, R. J., Jr. Cholinergic synapses in the lateral hypothalamus for the control of predatory aggression in the rat. Brain Research, 1970, 20, 409-424.

Bandler, R. J., Jr. Direct chemical stimulation of the thalamus: Effects on aggressive behavior in the rat. Brain Research, 1971 a, 26, 81-93.

Bandler, R. J., Jr. Chemical stimulation of the rat midbrain and aggressive behavior. New Biology, 1971b, 229, 222-223.

Bandler, R. J., Jr., \& Moyer, K. E. Animals spontaneousiy killed by rats. Communications in Behavior Biology, 1970, 5 , 177-182.

Karli, P. The Norway rat's killing response to the white mouse. Behavior, 1956, 10, 81-103.

Karli, P., Vergnes, M.. \& Didiergeorges, F. Rat-mouse interspecific aggressive behavior and its manipulation by brain ablation and brain stimulation. In E. B. Sigg and S. Garattini (Eds.), Biology of aggressive behavior. Amsterdam: Excerpta
Medica Foundation, 1969. Pp. 47-55.

King, M. B., \& Hoebel, B. G. Killing elicited by brain stimulation in rats. Communications in Behavioral Biology, 1968, 2, 173-177.

Moyer, K. E. Kinds of aggression and their physiological basis. Communications in Behavioral Biology, 1968, 2, 66-87.

Panksepp, J. Aggression elicited by electrical stimulation of the hypothalamus in albino rats. Physiology \& Behavior, 1971, 6, 321-329.

Smith, D. E King, M, B. \& Hoebel, B. G. Lateral hypothalamic control of killing: Evidence for a cholinoceptive mechanism. Science, 1970, 167, 900-901.

Vergnes, M., \& Karli, P. Effets de la stimulation de l'hy pothalamus lateral, de l'amygdale et de l'hippocampe sur le comportement d'agression interspecifique Rat-Souris. Physiology \& Behavior, 1969, 4, 889-894.

(Received for publication March 12, 1973.)

\section{Information processing in the visual periphery*}

\author{
JAMES R. ANTES and DAVID C. EDWARDS \\ Iowa State University, Ames, Iowa 50010
}

The information processing capacity of the visual periphery was investigated in two experiments using stimuli of known relative information content. The results of Experiment I showed redundant patterns to be easiest to identify at all peripheral angles tested $(5,10$, 20 , and $30 \mathrm{deg}$ ) as compared to intermediate and nonredundant patterns. Performance on all patterns decreased as peripheral angle increased. In Experiment II, simultaneous foveal and peripheral vision was required in a discrimination task. Performance was above chance only when the peripheral (7-deg) pattern was redundant. The foveal pattern had no effect on discrimination.

Many researchers attempting to describe visual information processing have dichotomized the regions of visual input into fovea and periphery. Typically they have characterized the foveal region as the source of almost all information input, ascribing only crude processing ability to the periphery (e.g., Gibson, 1966; Neisser, 1967; Williams, 1966). The bulk of the research, however, supports the notion of a structural and

*Portions of this research were reported at the 1970 Psychonomic Society meeting. functional continuum from the fovea to the extreme periphery. Polyak (1941) distinguished seven retinal regions based on changes within layers of the retina, emphasizing that these areas are not abruptly separated but blend into each other. Since the research of Wertheim (1894), many investigators have reported a continuous decrement in visual acuity with increasing peripheral angle. Numerous others have reported a similar decline in other measures of visual performance with peripheral stimulus presentation.

One important functional question concerns the relative efficacy of a dichotomous vs a continuous model of visual field in guiding future research on visual information processing. It appears certain that a more comprehensive model of visual search, for example, is possible if a continuum of visual field input is assumed. The research reported here is a preliminary investigation of peripheral information processing. Much is known about the acuity of the periphery and its capacity to detect geometric forms (e.g., Geissler, 1926; Menzer \& Thurmond, 1970). However, little is known about peripheral processing of stimuli varying on a redundancy dimension. Quantification of processing capabilities across the retina appears to be an important first step in modifying the model of visual field input. The following two experiments are initial attempts to investigate 